\title{
Effects of arousal level and below-zero habituation training on the spontaneous recovery and dishabituation of the orienting response
}

\author{
ROBERT J. GATCHEL, ELIZABETH GAAS, JAMES M. KING, \\ and MARK E. McKINNEY \\ University of Texas, Arlington, Texas 76019
}

\begin{abstract}
The effects of sympathetic arousal level on habituation and below-zero habituation of the skin conductance component of the orienting response (OR) were investigated in an experiment employing 60 subjects. Threat of shock was used to increase the arousal level of one group of subjects. These subjects were compared to a group that was not threatened by shock. Half of the subjects in each group received below-zero habituation training; the other half received no below-zero habituation. Results indicated that threat of shock significantly increased tonic skin conductance level. This increase in physiological arousal level was associated with decreased habituation of the OR. Moreover, increased arousal level and below-zero habituation training significantly affected the subsequent reappearance of the OR to a novel, dishabituating stimulus. The results of this study clearly demonstrate that physiological arousal level significantly affects the habituation and below-zero habituation processes.
\end{abstract}

In a recent study, Gatchel and Gaas (1976) investigated the effects of sympathetic arousal level on short- and long-term habituation of the skin conductance response component of the orienting response (OR). Threat of shock was used to increase the arousal level of one group of subjects. These subjects were compared to a group which was not threatened by shock. Findings of this experiment demonstrated that threat of shock significantly increased tonic skin conductance level. More importantly, this increase in physiological arousal level was associated with decreased short- and long-term habituation of the OR. These results clearly demonstrate that physiological arousal level significantly affects the habituation process.

The above results, as well as earlier findings by Gatchel (1975), suggest that a high level of physiological arousal may interfere with the consolidation process that prompts habituation. Indeed, the dualprocess theory advocated by Groves and Thompson (1970) assumes that habituation is a consequence of both phasic response and state processes. The results of the Gatchel and Gaas (1976) investigation demonstrate the importance of state processes, as indexed by sympathetic arousal level, in both short-term and long-term habituation.

The present experiment was designed to examine whether elevation of sympathetic arousal level also

This research was supported in part by a grant to the first author from the University of Texas at Arlington Organized Research Fund. significantly affects the "below-zero" habituation process. A number of experiments investigating human ORs have demonstrated that below-zero habituation, that is, the continuation of the habituation process beyond the point at which zero responding has occurred, did not result in less spontaneous recovery of the OR after some recovery period, relative to the administration of no additional belowzero habituation training (e.g., Stephenson \& Siddle, 1976; Waters \& McDonald, 1974). These findings are contrary to predictions based on the Groves and Thompson (1970) model, which would predict less spontaneous recovery associated with greater belowzero habituation training. An important question requiring investigation is whether the direct manipulation of the state-arousal level significantly influences the effects of this below-zero habituation process. These data should further illuminate the importance of physiological arousal level on the short- and long-term habituation process. Its effect on the dishabituation process will also be evaluated.

\section{METHOD}

\section{Subjects}

The subjects consisted of 60 undergraduates from the introductory psychology subject pool at the University of Texas at Arlington. They received points to be applied to their course grade for participation in this experiment. All subjects reported having normal hearing.

\section{Apparatus}

Skin resistance was continuously monitored throughout the experiment on a Narco Bio-Systems Physiograph. Beckman silver- 
silver chloride electrodes ( $1-\mathrm{cm}$ radius) were attached to the palm and ventral side of the wrist of the subject's nonpreferred hand to record skin resistance. An inactive, "dummy" shock electrode was attached to the wrist of the subject's preferred hand. Johnson \& Johnson K-Y jelly was used as the electrolyte in recording skin resistance. A Physiograph GSR coupler imposed a current of $10 \mu \mathrm{A}$ through the two electrodes. Two channels of electrodermal data were collected. The first channel was dc-coupled and recorded tonic skin-resistance levels at a low sensitivity setting (2,500-ohm resistance change sensitivity). The second channel was capacitor-coupled, with a 6-sec time constant, which allowed the recording of short-term phasic responses at a high sensitivity setting (500-ohm resistance change sensitivity).

Stimuli were 2 -sec tones presented binaurally over a matched set of Grason-Stadler earphones. The tones were generated by a Hewlett-Packard $200 \mathrm{CD}$ oscillator, with a ramp-control electronic switch imposing a $10-\mathrm{msec}$ rise and decay time on the tones. The tone intensity was $65 \mathrm{~dB}$, as measured by scale Cs of a General Radio Company sound-level meter. For habituation trials, tone frequency was set at $1,000 \mathrm{~Hz}$. For dishabituation, tone frequency was $500 \mathrm{~Hz}$. The 2 -sec tone presentation was controlled by a Scientific Prototype interval timer.

\section{Experimental Design and Procedure}

Subjects were randomly assigned to one of four groups (15 subjects per group). Subjects in Groups 1 and 2 received threatof-shock instructions, to be described below, in Part 1 of the experiment. In this part of the experiment, they received tones every 25,30 , or $35 \mathrm{sec}$, with a mean ISI of $30 \mathrm{sec}$, until they reached the criterion for habituation, which was defined as five consecutive stimulus presentations with no skin resistance response of $500 \mathrm{ohms}$ or more within $1-5 \mathrm{sec}$ following stimulus onset. Group 2 subjects then received 10 additional stimulus presentations (below-zero habituation training). Groups 3 and 4 were identical to Groups 1 and 2, respectively, except for the fact that subjects in these former two groups did not receive threat-of-shock instructions. Thus, the four groups were: Group 1 (threat-of-shock/no below-zero habituation training), Group 2 (threat-of-shock/below-zero habituation training), Group 3 (no threat-of-shock/no below-zero habituation training), Group 4 (no threat-of-shock/below-zero habituation training).

At the start of Part 1 of the experiment, the subject was seated in a comfortable chair in a partially soundproof room. After the recording electrodes were attached, the experimenter calibrated the recording equipment in an adjacent room. The experimenter then read the differential group instructions. Groups 3 and 4 were administered instructions which informed them that they would hear a series of tones. Groups 1 and 2 received similar instructions, but with the addition of a threat-of-shock statement. The instructions are presented below. Group 3 and Group 4 subjects were not read the sentence within the parentheses. This sentence, intended to elicit an elevation of sympathetic arousal, was administered only to the Group 1 and Group 2 subjects: "During the course of the experiment you will be presented some tones through the headset. (Also, you may receive a mild electric shock through the electrode on your wrist at some point during the experiment.) Please sit quietly, relax but do not fall asleep. Please try to move as little as possible since movement interferes with our physiological recording. I will let you know over the intercom when the session is completed. Do you have any questions? If not, we will get started in a few minutes."

After reading the instructions, the experimenter left the room and, after a 10-min baseline period, presented the stimuli. It should be noted that at no time during the experiment was shock ever administered to the subjects. At the completion of Part 1 of the experiment, the subjects were read another set of instructions. These instructions, presented below, introduced Part 2, and were intended to eliminate the threat of shock in the Group 1 and Group 2 subjects. Again, the sentence within the parentheses was not read to Groups 3 and 4: "During this second part of the experiment, you will again be presented some tones through the headset. (You do not, however, have to worry about receiving an electric shocking during this part of the experiment.) Again, please sit quietly, relax but do not fall asleep. Please move as little as possible since movement interferes with our physiological recording."

The "dummy" shock electrode was then removed from the wrist of all subjects. A 15-min rest period was then given, after which Part 2 of the experiment was administered. In Part 2 of the experiment, which tested for long-term habituation effects, tones were again administered to all subjects, with a mean ISI presentation rate of $30 \mathrm{sec}$, until they reached the criterion for habituation, which was defined as three consecutive stimulus presentations with no skin resistance response. At the completion of Part II, the subjects received one presentation of a dishabituation tone $(65 \mathrm{~dB}, 500 \mathrm{~Hz})$.

\section{Data Reduction and Analysis}

Phasic skin-resistance response measures were transformed to units of $\log$ conductance using the formula: $\log \left(1 / R_{1}\right)-\log$ $\left(1 / R_{2}\right)$, where $R_{1}$ is the maximum resistance deflection peak occurring within $5 \mathrm{sec}$ of stimulus onset, and $R_{2}$ is the prestimulus resistance base level. Tonic skin resistance levels were measured prior to the onset of each stimulus and converted to conductance units.

The skin resistance data were measured by scorers who had no knowledge of the experimental contingencies of the present study. The data were punched on computer cards for computation of the above-described transformations and later statistical analyses on an IBM 370 computer.

\section{RESULTS}

Analyses of variance were performed on the skin conductance base level data (base levels prior to the first tone presentation) for Parts 1 and 2. Based upon the results of the Gatchel and Gaas (1976) study, a number of planned contrasts of group means were performed. These contrasts indicated that the two threat-of-shock instruction groups (Groups 1 and 2) had significantly higher tonic skin conductance levels during both Part $1, \mathrm{~F}(1,56)=6.86, \mathrm{p}<.025$, and Part 2, $\mathrm{F}(1,56)=5.38, \mathrm{p}<.05$, relative to the nonthreatened group subjects. This effect of threat-ofshock instructions on tonic level is similar to that found in the Gatchel and Gaas (1976) study.

Average phasic skin conductance response curves for the four groups, plotted for the first five trials of Part 2, are presented in Figure 1. Only the first five trials are plotted because of the differing number of tone presentations received by subjects during Part 2 due to the habituation criterion employed; all subjects, however, received at least five stimulus presentations. A Kruskal-Wallis analysis of variance by ranks was conducted on the group averages of the five trials. This analysis revealed a significant group effect, $\chi^{2}(3)=10.13, p<.025$. Mann-Whitney tests for equality of group means indicated that Group 2 (threat-of-shock instructions/below-zero habituation) was significantly different from Group 1, $\mathrm{z}=2.01, \mathrm{p}<.05$, Group $3, \mathrm{z}=2.63, \mathrm{p}<.01$, and Group $4, \mathrm{z}=2.43, \mathrm{p}<.01$. Also, Group 1 (threat-of-shock instructions/no below-zero habitua- 
Table 1

Average Skin Conductance Response Magnitudes for Each Group Elicited by the Dishabituation Trial

\begin{tabular}{lc}
\hline & $\begin{array}{c}\text { Average Response } \\
\text { Magnitudes }\end{array}$ \\
\hline Group 1 (threat of shock/no below-zero habituation) & .0284 \\
Group 2 (threat of shock/below-zero habituation) & .0413 \\
Group 3 (no threat of shock/no below-zero habituation) & .0216 \\
Group 4 (no threat of shock/below-zero habituation) & .0261 \\
\hline
\end{tabular}

tion) was near-significantly different from Group 3, $\mathrm{z}=1.83, \mathrm{p}<.10$, and Group $4, \mathrm{z}=1.66, \mathrm{p}<.10$. These analyses confirm the group differences that may be seen in Figure 1, with Group 2 showing the greatest responding at the start of Part 2, i.e., less long-term habituation. Groups 3 and 4 demonstrated the greatest amount of long-term habituation.

Table 1 lists the mean skin conductance response for each group elicited by the dishabituation test trial. A Kruskal-Wallis analysis of variance of these data yielded a significant group effect, $\chi^{2}(3)=12.41$, $\mathrm{p}<.01$. Mann-Whitney tests indicated that Group 2 was significantly different from Group $1, \mathrm{z}=2.55$, $\mathrm{p}<.01$, Group 3, $\mathrm{z}=2.25, \mathrm{p}<.01$, and Group 4, $\mathrm{z}=2.41, \mathrm{p}<.01$. Thus, as can be seen in Table 1 , Group 2 demonstrated the greatest OR to the novel dishabituating stimulus.

\section{DISCUSSION}

The results of the present investigation replicate

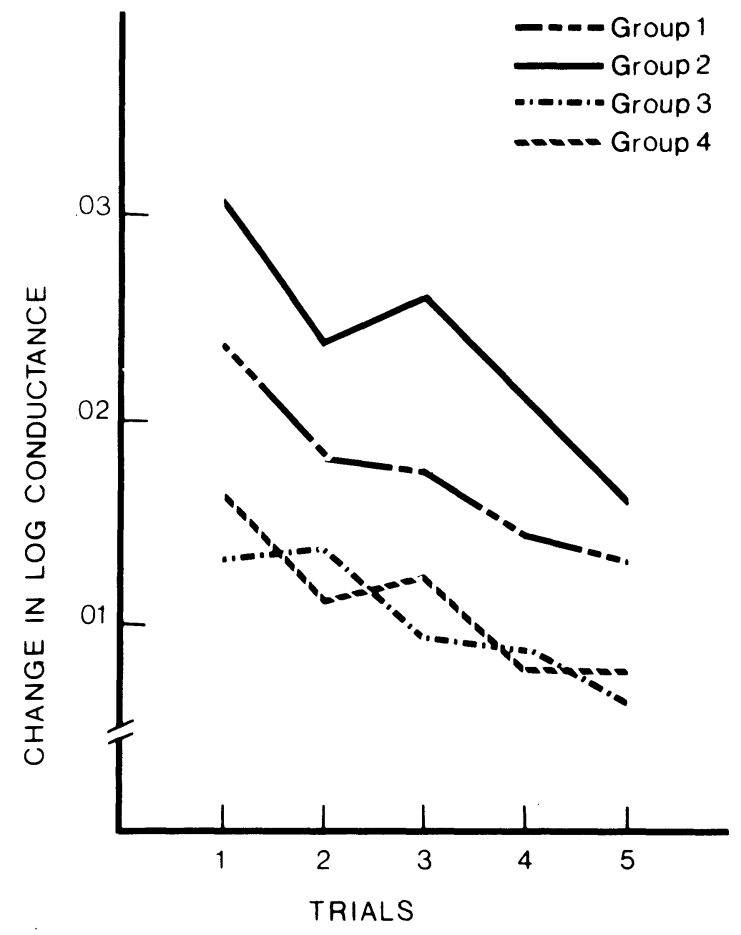

Figure 1. Average skin-conductance responses for groups for the first five trials of Part 2. the previously reported findings of Gatchel and Gaas (1976) in demonstrating that arousal level significantly affects long-term habituation of the electrodermal component of the OR. Subjects who received threatof-shock instructions in Part 1 of the experiment displayed significantly higher tonic skin conductance levels relative to subjects in the nonthreatened groups. These same subjects demonstrated less habituation when tested in Part 2 for long-term habituation.

The major new finding of the present study was the demonstration that arousal level significantly affects the below-zero habituation process. In Part 2 of the experiment, Group 2 subjects who had previously received threat-of-shock instructions and below-zero habituation training demonstrated greater spontaneous recovery of the OR (i.e., less long-term habituation) relative to the Group 1 subjects who received below-zero habituation training but no threat-of-shock instructions. Results also demonstrated that increased arousal level and belowzero habituation training significantly affects the subsequent reappearance of the OR to a novel, dishabituating stimulus. The threat-of-shock/belowzero habituation group demonstrated the greatest magnitude of responding when the dishabituation test trial was administered. Waters and McDonald (1974) had earlier reported that subjects receiving below-zero habituation training displayed significantly larger ORs to a dishabituating stimulus than those subjects who received no such training. The present results indicate that increased arousal level in combination with below-zero habituation training produces an even greater reemergence of responding during dishabituation.

The findings of the present investigation, in agreement with results reported by Gatchel and Gaas (1976), again indicate that a high level of physiological arousal can interfere with the consolidation process that prompts habituation. As indicated in this earlier study, the results clearly demonstrate the importance of state processes, as indexed by sympathetic arousal level, in long-term habituation. The dual-process theory advocated by Groves and Thompson (1970) assumes that habituation is a consequence of both phasic response and state processes. Present results also demonstrate the importance of state processes in determining the degree of responding to a dishabituating stimulus. 


\section{REFERENCES}

GATCHEL, R. J. Effects of interstimulus interval length on shortand long-term habituation of autonomic components of the orienting response. Physiological Psychology, 1975, 3, 133-136.

GATCHEL, R. J., \& GAAs, E. Effects of arousal level on short- and long-term habituation of the orienting response. Physiological Psychology, 1976, 4, 66-68.

Groves, P. M., \& Thompson, R. E. Habituation: A dual-process theory. Psychological Review, 1970, 77, 419-450.
Stephenson, D., \& Siddle, D. A. T. Effects of "below-zero" habituation on the electrodermal orienting response to a test stimulus. Psychophysiology, 1976, 13, 10-15.

Waters, W. F., \& McDonald, D. G. Effects of "below-zero" habituation on spontaneous recovery and dishabituation of the orienting response. Psychophysiology, 1974, 11, 548-558.

(Received for publication January 21, 1977; accepted February 7, 1977.) 